\title{
Genome editing and transcriptional repression in Pseudomonas putida KT2440 via the type II CRISPR system
}

\author{
Jun Sun', Qingzhuo Wang ${ }^{2}$, Yu Jiang ${ }^{2,3}$, Zhiqiang Wen², Lirong Yang ${ }^{1}$, Jianping Wu ${ }^{1 *}$ (D) and Sheng Yang 2,3,4*
}

\begin{abstract}
Background: The soil bacterium Pseudomonas putida KT2440 is a "generally recognized as safe"-certified strain with robust property and versatile metabolism. Thus, it is an ideal candidate for synthetic biology, biodegradation, and other biotechnology applications. The known genome editing approaches of Pseudomonas are suboptimal; thus, it is necessary to develop a high efficiency genome editing tool.

Results: In this study, we established a fast and convenient CRISPR-Cas9 method in P. putida KT2440. Gene deletion, gene insertion and gene replacement could be achieved within 5 days, and the mutation efficiency reached $>70 \%$. Single nucleotide replacement could be realized, overcoming the limitations of protospacer adjacent motif sequences. We also applied nuclease-deficient Cas9 binding at three locations upstream of enhanced green fluorescent protein (eGFP) for transcriptional inhibition, and the expression intensity of eGFP reduced to $28.5,29.4$, and $72.1 \%$ of the control level, respectively. Furthermore, based on this CRISPR-Cas9 system, we also constructed a CRISPR-Cpf1 system, which we validated for genome editing in P. putida KT2440.
\end{abstract}

Conclusions: In this research, we established CRISPR based genome editing and regulation control systems in $P$. putida KT2440. These fast and efficient approaches will greatly facilitate the application of P. putida KT2440.

Keywords: CRISPR-Cas9 system, Pseudomonas putida KT2440, Genome editing, Single nucleotide mutation, Transcriptional engineering, CRISPR-Cpf1 system

\section{Background}

Pseudomonas putida KT2440 is an environmentally safe, non-pathogenic Pseudomonad species [1]. Because of its outstanding capacity to degrade aromatics compounds and robust viability in harsh conditions, $P$. putida KT2440 is an ideal chassis for bioremediation [2], metabolic engineering [3], and biocatalysis [4]. Several genome editing approaches have been applied into Pseudomonas. A series of molecular tools, such as counter-selection markers (sacB [5], pyrF [6], upp [7]), heterologous recombinases (Cre-loxP [8], Flp-FRT [9]), and suicide vectors

\footnotetext{
*Correspondence: wjp@zju.edu.cn; syang@sibs.ac.cn

1 Institute of Bioengineering, College of Chemical and Biological Engineering, Zhejiang University, Hangzhou 310027, China

${ }^{2}$ Key Laboratory of Synthetic Biology, Institute of Plant Physiology and Ecology, Shanghai Institutes for Biological Sciences, Chinese

Academy of Sciences, Shanghai 200032, China

Full list of author information is available at the end of the article
}

[10] have been validated and spurred the advancement of allelic exchange for engineering mutations in this genus. Bacteriophage-based recombination proteins (Red/ET $[11], \lambda$-Red $[12,13])$ enable homologous recombination between a target genome locus and donor DNA, which is an efficient method in Pseudomonas aeruginosa PAO1 [12] and P. putida KT2440 [11, 13]. Based on homologous recombination in double-stranded breaks (DSB), the I-SceI homing endonuclease has been developed as a seamless genome editing tool in P. putida KT2440 [14]. In another chromosomal engineering approach used in Pseudomonas, transposon vectors-based methods [1517] have excellent transposition frequencies. Although these various strategies have been applied into genome engineering of Pseudomonas, they still have many drawbacks, such as time-consuming manipulation, scars left 
in the genome, inability to target exact loci, difficulty in generating mutants, and deficiency in plasmid-curing.

Clustered Regularly Interspaced Short Palindromic Repeats (CRISPR)-Cas (RNA-guided proteins) systems are a prokaryotic adaptive immune defense mechanism in many bacteria and most archaea [18]. Among three major types, the type II CRISPR-Cas system from Streptococcus pyogenes (SpCas9) is the best characterized [19]. In the CRISPR-Cas9 system, a chimeric single guide RNA (sgRNA) is used for Cas9 sequencespecified guidance [20], and a short protospacer adjacent motif (PAM) exerts recognition between Cas9 and the target DNA [21]. Cas9 protein then catalyzes the breaking of DNA double strands [22]. The DSB caused by Cas9 can be repaired via homology-directed repair (HDR) [23] or non-homologous end joining (NHEJ) [24] in eukaryotes. Cas9-nickase (nCas9), in which one domain is inactivated (D10A or H840A variant), can reduce the lethal effect on cells and the repairing process can occur more easily $[25,26]$. A nucleasedeficient Cas9 (dCas9) retains sgRNA binding ability and can be applied for target gene regulation, including blocked transcription for CRISPR-based interference [27], or fused with activators for RNA-guided activation [28]. Because of its marker-free, cost-efficient, simple genome manipulation, the CRISPR-Cas9 system has been developed as a genome engineering tool in a wide range of prokaryotes and eukaryotes, including (but not limited to) Escherichia coli [29], Clostridium cellulolyticum [30], Streptomyces species [31], Bacillus subtilis [32], Yarrowia lipolytica [33], Saccharomyces cerevisiae [34], mammalian cells [35], and zebrafish [36]. Recently, Aparicio et al. [37] developed a CRISPR/ Cas9-based three-plasmid system in $P$. putida, and gene deletion or single nucleotide substitution can be achieved. However, this three-plasmid system has challenges regarding plasmid-curing, especially for continual genome editing, and other versatile characteristics of type II CRISPR system have not been fully explored in P. putida KT2440.

Cpf1 is a newly discovered single RNA-guided nuclease [38] that belongs to type V-A CRISPR systems [39]. In contrast with the chimeric sgRNA-associated Cas9, in the CRISPR-Cpf1 system, Cpf1 recognizes 'TTN' PAM sequence. The PAM sequence is located at the $5^{\prime}$ - end of the target sequence, and the spacer sequence follows a 19-nt CRISPR RNA (crRNA) direct repeat. Aside from the different recognition mechanism compared with Cas9, the staggered cutting style of Cpf1 could facilitate gene insertion for NHEJ repair mechanisms [38]. Taken together, these characteristics make CRISPR-Cpf1 an attractive complementary tool to the well-established CRISPR-Cas9 system.
Because of the versatile functions and simple manipulation of CRISPR-Cas9, we developed a CRISPR-Cas9 based genome editing system to overcome major limitations of the known gene editing technologies of $P$. putida KT2440. Here, we tested Cas9 toxicity and analyzed sgRNA off-target effect; multiple genes were targeted for integration or deletion by our two-plasmid system. Catalytically inactive Cas9 (dCas9) was applied to control gene expression via transcriptional repression. Additionally, we preliminarily explored the CRISPR-Cpf1 system for genome editing in P. putida KT2440. These systems could be powerful tools to extend the application of $P$. putida KT2440, and pave the way for use of CRISPR systems into other Pseudomonads.

\section{Methods}

\section{Strains, culture conditions and reagents}

The strains and plasmids used in this study are given in Additional file 1. All kinds of E. coli, and P. putida KT2440 were grown in LB medium (Liquid and Solid). E. coli DH5 $\alpha$ was used for all cloning and maintenance, while E. coli S17-1 served as a helper strain for conjugal transfer. E. coli were grown at $37{ }^{\circ} \mathrm{C}$, and $P$. putida KT2440 was incubated at $30{ }^{\circ} \mathrm{C}$. Antibiotics were added at the following concentrations: kanamycin, $150 \mu \mathrm{g} / \mathrm{mL}$ (50 $\mu \mathrm{g} / \mathrm{mL}$ for E. coli); gentamicin, $50 \mu \mathrm{g} / \mathrm{mL}$; tetracycline, $25 \mu \mathrm{g} / \mathrm{mL}(15 \mu \mathrm{g} / \mathrm{mL}$ for $E$. coli) and spectinomycin, $100 \mu \mathrm{g} / \mathrm{mL}$.

The DNA polymerase PrimeSTAR ${ }^{\circledR}$ Max, all restriction endonucleases and T4 DNA ligase were purchased from Takara Bio Inc (Dalian, China). The One Step Cloning Kit (Vazyme Biotech Co., Ltd, Nanjing, China) and pEASYUni Seamless Cloning and Assembly Kit (TransGen Biotech, Beijing, China) were applied for seamless cloning.

\section{Plasmids construction and N20 sequence selection}

The CRISPR/Cas9 system consists of two plasmids: pCAS-RK2K and pSEVA-gRNAT (Fig. 1). Plasmid pCAS-RK2K was constructed from plasmid pCASsac (an unpublished E. coli CRISPR/Cas9 2.0 system), which was a gift from Yang Sheng. Plasmid pSEVA-gRNAT was derived from pTargetF [29] and pSEVA644, the latter together with its same series of plasmids was obtained from SEVA Database [40]. All primers used in this study are listed in Additional file 2.

Based on pCAS backbone [29], the construction of pCASsac focused on the following steps: a tightly-controlled Rhas-PrhaB promoter replaced with lacIq-Ptrc promoter; pSC101 replicon as a substitute for the temperature-sensitive replicon repA101 (Ts); SacB acted as a curing component was inserted into the plasmid. In our study, two broad-host-range replicons, RK2 (together with oriT fragment) from pSEVA429 and pBBR1 from 


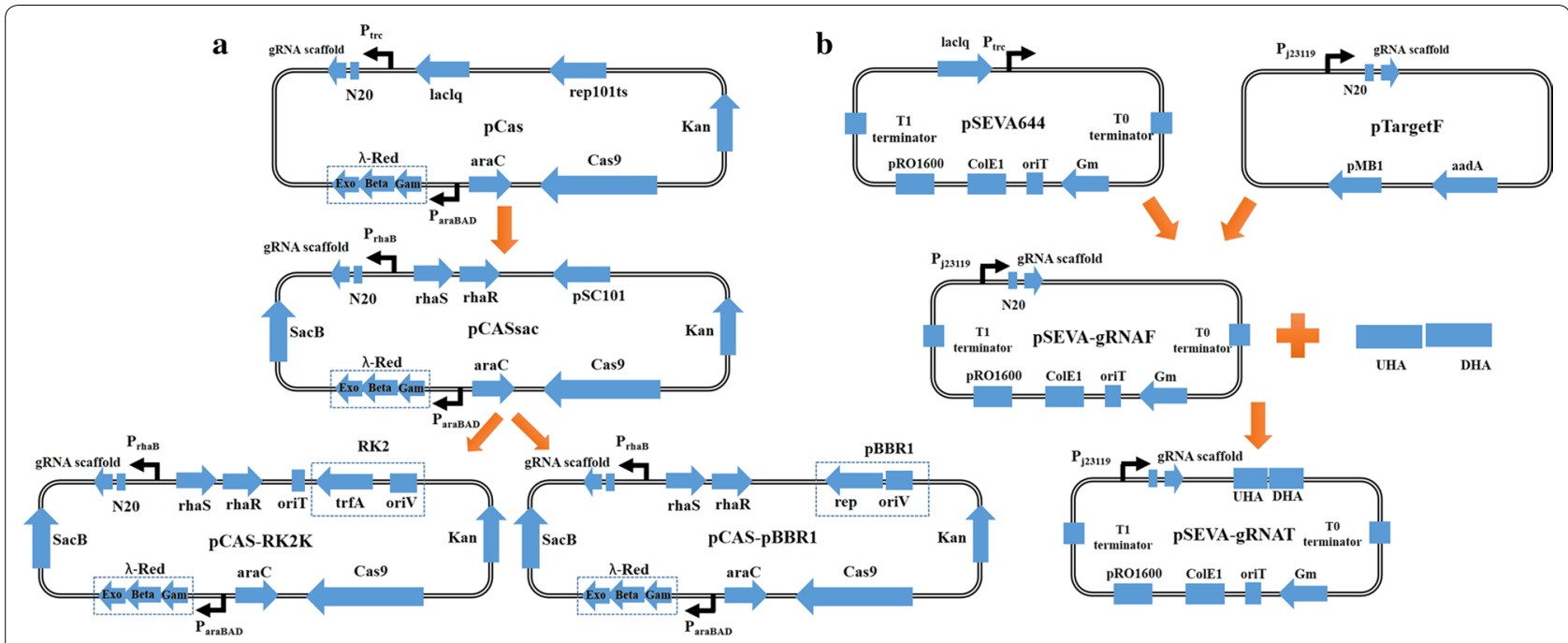

Fig. 1 Strategy for the construction of a CRISPR-Cas9 two-plasmid system in P. putida KT2440. a pSC101 replicon in pCASsac was replaced with RK2 replicon together with oriT fragment, creating pCAS-RK2K. In pCAS-RK2K, cas9 gene was linked with its native promoter, and gRNA cassettes were transcribed by $P_{\text {rhaB }}$ promoter so as to guide Cas 9 protein targeting $p R O 1600$ replicon in PSEVA-gRNAT. The $\lambda$-Red recombination system was under control of arabinose promoter to enhance the repairing efficiency in KT2440. SacB, a commonly used counterselectable marker function as a self-curing tool. $\mathbf{b}$ The $P_{t r c}-1 a c 1^{19}$ inducible system was eliminated from pSEVA644 and gRNA cassettes were inserted, generating pSEVA-gRNAF. The upstream homologous arm (UHA) and downstream homologous arm (DHA) were amplified from genome and connected by overlap-extension PCR. Next, the combinational homologous arm was assembled into PSEVA-gRNAF, giving rise to PSEVA-gRNAT

pBBR1MCS2 were amplified using primers Sa-RK-1F/SaRK-2R and Sa-BBR1-1F/Sa-BBR1-2R, respectively. The replication origin of pCASsac was then replaced with RK2 or pBBR1 using the One Step Cloning Kit (the detailed construction strategies are shown in Additional file 3), thus creating plasmids pCAS-RK2K and pCAS-pBBR1 (Fig. 1a), respectively. pCAS-RK2K has a tetracycline version: pCAS-RK2T (the kanamycin resistance marker in pCAS-RK2K was replaced with a tetracycline resistance marker). Elimination of Cas9 or $\lambda$-Red inducible expression system from pCAS-RK2K respectively, pCASRK2K $\triangle$ Cas9 and pCAS-RK2K $\triangle$ Red were constructed via Gibson assembly with primers $\mathrm{QC}-1 \mathrm{~F} / \mathrm{QC}-1 \mathrm{R}, \mathrm{XC}-2 \mathrm{~F} /$ $\mathrm{XC}-2 \mathrm{R}$ and JH-1F/JH-1R, JH-2F/JH-2R, JH-3F/JH-3R.

Plasmid pSEVA-gRNAT (Fig. 1b) was assembled from the following fragments: LacIq-ptrc promoter was removed from pSEVA644, and then pSEVA644 $\triangle$ LacIqptrc was used as backbone; $j 23119$ promoter and sgRNA cassette from pTargetF were fused into the EcoRI/SacI digested pSEVA644 $\triangle$ LacIq-ptrc via primers JS-1F/ JS-2R. We selected nicC (Locus tag is PP_3944) as target site, which is an unessential gene in the P. putida KT2440 genome. pSEVA-gRicF was derived from pSEVA-gRNAT by replacing the original N20 sequence with 'AAAATC GCAATCGTCGGTGC' via inverse PCR using primers NC-F/NC-R. The homologous arms of nicC were acquired from the Pseudomonas Genome Database [41]. Next, 500 bp length upstream and downstream of the target gene were amplified using primers NT-1F/NT-2R, NT-ZF/NT-ZR, and connected via overlap-extension (SOE) PCR. The resulting homologous arm was then inserted into the BamHI/HindIII-digested pSEVA-gRicF, thus creating pSEVA-gRicT.

Here, the N20 sequence of low off-target rate was designed via CasOT [42]. N20 sequences of all pSEVAgRNAT derivatives were constructed by two reverse primers with $20 \mathrm{nt}$ reverse complementarity. The repairing homologous arms were ligated into pSEVA-gRNAT by BamHI and HindIII sites or using Gibson assembly. Toward different DNA template strand of nicC (PP_3944), pSEVA-gRic6T and pSEVA-gRic5T were constructed from pSEVA-gRicT via primers Nic6F/ Nic6R and Nic5F/Nic5R, respectively. The target-specificity of pSEVA-gRic6T and pSEVA-gRic5T was calculated by CasOT. Due to its specificity in the genome, the N20 sequence ('CATTCAGAACTAACTTGTCG') was inserted into pSEVA-gRNAT via primers DgRNA-F/ DgRNA-R, thus creating pSEVA-dgRNA. pSEVA-dgRNA was designed as a positive control. The sequence 'GGT TGTAGGAAGATTCGATA' from pSEVA-gRNAT replicon (pRO1600) was selected as N20 sequence under the transcriptional control of rhamnose-inducible promoter (Rha), in which pCAS-RK2K was applied to cure pSEVAgRNAT. All of the N20 sequences designed by CasOT and primers used for pSEVA-gRNAT derivatives are listed in Additional files 2 and 4. 


\section{Plasmid transformation method}

Plasmid pCAS-pBBR1, pCAS-RK2K, pCAS-RK2T, pSEVA-gRNAT or other derivatives was transformed into $P$. putida KT2440 by electroporation. Firstly, KT2440 was inoculated and cultivated overnight, then transferred to a $100 \mathrm{~mL}$ flask containing $10 \mathrm{~mL} \mathrm{LB}$ without antibiotics until cell densities $\left(\mathrm{OD}_{600}\right)$ reached 0.6-0.8. After concentration, the electrocompetent cells were prepared by three times washing of $3 \mathrm{mM}$ HEPES Buffer [13], then concentrated to $500 \mu \mathrm{L}$. For electroporation conditions, $100 \mu \mathrm{L}$ bacterial suspension and $150 \mathrm{ng}$ DNA were transferred to a $2 \mathrm{~mm}$ gap ice-cold electroporation cup and the detailed setting of Bio-Rad GenePulser II were listed as follows: $2.5 \mathrm{kV}$; $200 \mathrm{~V} ; 25 \mathrm{uF}$. After electroporation, $1 \mathrm{~mL}$ LB liquid medium was added and mixed, then the entire mixture was transferred into a $2.0 \mathrm{~mL}$ Eppendorf tube and cultivated in $30{ }^{\circ} \mathrm{C}$ for $2 \mathrm{~h}$ before being spread on $\mathrm{LB}$ selection plates.

Alternatively, E. coli S17-1 could be used as a helper strain to transfer pCAS-RK2K or its derivatives into $P$. putida KT2440. E. coli S17-1 containing plasmid (adding related antibiotics), and Pseudomonas species were cultivated in LB medium overnight, then each bacteria was transferred to a $5 \mathrm{~mL} \mathrm{LB}$ tube (without antibiotics) for incubation until the $\mathrm{OD}_{600}$ reached 0.6-0.8. All these cells were concentrated and washed four times with sterile water, then $E$. coli mixed with Pseudomonas in a ratio of 1:1. Next, these mixtures were dripped on LB agar plates in small dots, and incubated at $37^{\circ} \mathrm{C}$ for $6 \mathrm{~h}$, then shifted to $30{ }^{\circ} \mathrm{C}$ overnight. After incubation, the mixture cells were scraped from the plate, and diluted and spread on selection plates (Tetracycline $25 \mu \mathrm{g} / \mathrm{mL}$ or kanamycin $150 \mu \mathrm{g} / \mathrm{mL}$, together with spectinomycin $100 \mu \mathrm{g} / \mathrm{mL}$ ) for $12-16 \mathrm{~h}$ at $30^{\circ} \mathrm{C}$.

\section{Toxicity analysis}

As the catalytic activity of Cas9 influences its toxicity in several prokaryotic microbes, we constructed four versions of Cas9 by introducing point mutations at the D10A, H840A, D10A and H840A together, as well as frameshift mutation in the start codon by transforming ATG to AGTG (Cas9FM) via primers PDF1F/ PDF1R, PDF2F/PDF2R, and PDFM-1F/PDFM-1R, respectively. Each Cas9 derivative was assembled into pCAS-RK2K $\triangle$ Cas9, creating the following plasmid pCAS-RK2K-nCas9D, pCAS-RK2K-nCas9H, pCASRK2K-dCas9, and pCAS-RK2K-Cas9FM. In this experiment, pCAS-RK2K was used as a control. Using the same electroporation conditions, $150 \mathrm{ng}$ of these plasmids were transformed into $P$. putida KT2440 in respective triplicate, and then screened by kanamycin plates. After cultivated at $30{ }^{\circ} \mathrm{C}$ for $18 \mathrm{~h}$, the average number of transformants was calculated as $\mathrm{CFU} / \mu \mathrm{g}$ of DNA.

\section{Genome editing}

In this two-plasmid system, large size plasmid pCASRK2K or pCAS-RK2T was first transformed into $P$. putida KT2440 by electroporation or conjugal transfer.

After preparation of KT2440 harboring PCAS-RK2K or pCAS-RK2T, candidate colony was inoculated in LB liquid medium and cultivated overnight. Then overnight culture was transferred into $10 \mathrm{~mL}$ tube by $4 \%$, and $0.6 \%$ L-arabinose ( $0.06 \mathrm{~g}$ in $10 \mathrm{~mL}$ LB liquid) was added into the medium and completed cultivation about $2-2.5 \mathrm{~h}$. The bacteria cells were then harvested and washed by $3 \mathrm{mM}$ Hepes Buffer [13], and finally concentrated to $500 \mu \mathrm{L}$ for electroporation. Using the electroporation protocols described above, 150 ng of pSEVA-gRNAT or its derivatives was added. After the recovery process, $100 \mu \mathrm{L}$ final concentrated cells were plated on selection plates (Tetracycline $25 \mu \mathrm{g} / \mathrm{mL}$ or kanamycin $150 \mu \mathrm{g} / \mathrm{mL}$, together with gentamycin $50 \mu \mathrm{g} / \mathrm{mL}$ ) and cultivated at $30{ }^{\circ} \mathrm{C}$. Transformants were analyzed by colony PCR and confirmed by DNA sequencing.

In order to investigate the deficiency effect of four essential sections (Cas9, gRNA cassette, homologous repairing template, and $\lambda$-Red system) in this system, we designed a series of control experiment to calculate the total CFU and analysed the mutation efficiency. Plasmid pCAS-RK2K, pCAS-RK2K $\triangle$ Cas9 and pCASRK2K $\triangle$ Red (plasmid construction see above) were first transformed into P. putida KT2440, respectively. After KT2440 harboring the related PCAS-RK2K or its derivatives, pSEVA644, pSEVA-dgRNA, pSEVA-gRic6T, and pSEVA-gRic6F (derived from pSEVA-gRic6T by deletion of homologous repairing template) were sequential transformed into these strains, respectively (see below Fig. 3a).

\section{Plasmid curing}

After the identification procedure, the mutated colony was inoculated into a $5 \mathrm{~mL}$ LB medium with kanamycin $(150 \mu \mathrm{g} / \mathrm{mL})$ or tetracycline $(25 \mu \mathrm{g} / \mathrm{mL})$ plus rhamnose $(10 \mathrm{mM})$ and shaken at $30{ }^{\circ} \mathrm{C}$ overnight. Subsequently, the colonies were streak on LB selection plates containing kanamycin $(150 \mu \mathrm{g} / \mathrm{mL})$ or tetracycline $(25 \mu \mathrm{g} / \mathrm{mL})$, and confirmed as pSEVA-gRNAT lost by colony PCR via primers PS1/PS2. After the first round of genome editing, the pSEVA-gRNAT cured strain could be used in the next round of genome editing. Finally, a plasmid-cured colony was obtained by cultivated overnight with glucose ( $5 \mathrm{~g} / \mathrm{L})$, then diluted and streak in LB agar plates adding sucrose $(10 \mathrm{~g} / \mathrm{L})$ and glucose $(5 \mathrm{~g} / \mathrm{L})$. The curing of pCAS-RK2K was confirmed by colony PCR via primers $\mathrm{Ra}-J \mathrm{~J} / \mathrm{Ra}-J \mathrm{R}$ or identified its antibiotics sensitivity. 


\section{Single nucleotide mutation}

The construction of single nucleotide mutation in the genome can be divided into two strategies.

In the first strategy, single nucleotide mutation aimed to change the PAM sequence (a 3-nt upstream of N20 sequence for pSEVA-gRic6T), the nucleotide sequence 'CGG' to 'CAG' within nicC gene. We amplified the first $500 \mathrm{bp}$ of $n i c C$ and its upstream 500 bp length sequence from genome by primers NCD1F/NCD2R. Based on pSEVA-gRic6T backbone, we assembled the fragment into the plasmid (creating pSEVA-gRic6PAM1) via Gibson assembly and then site-directed mutation in 'CGG' was performed by primers NPAM-F/NPAM-R, generating pSEVA-gRic6PAM2. After electroporation of pSEVAgRic6PAM2 into KT2440 harboring pCAS-RK2K, the mutated colonies were amplified via identification primers D1-JF/D1-JR and confirmed by DNA sequencing.

In another strategy, we attempted to mutate 'CAA' to 'CTA' at position Gln139 in nicC gene. We developed a scarless two-step single nucleotide mutation strategy (Additional file 5). Firstly, we added 20 bp sequence 'ATG TCTCATAAGATCATTAC' (named A20 sequence and off-target should be avoided) between N20 sequence of pSEVA-gRic6PAM1 and its PAM sequence via inverse PCR using primers A20F/A20R. After the construction process and gene sequencing, we mutated the target single nucleotide on the homologous arm using primers Nic-SF/Nic-SR, thus creating pSEVA-NicA20. Another plasmid pSEVA-NicA21 was derived from pSEVANicA20 by deletion of A20 sequence in homologous arms and replaced the original N20 sequence with A20 sequence using primers NT20-F/NT20-R and NT21-F/ NT21-R, respectively. After the first-step editing process, pSEVA-NicA20 was cured from the colony and another plasmid pSEVA-NicA21 was then transformed in the second step. Finally, the mutated colonies were confirmed by DNA sequencing.

\section{Regulation of eGFP expression intensities by dCas9}

A catalytically deficient Cas9 was achieved by point mutations at two domains (D10A and H840A). For analysis of gene repression by $\mathrm{dCas} 9$, the relative fluorescence intensities of enhanced green fluorescent protein (eGFP) were measured by microplate reader (Thermo Varios$\mathrm{kan}^{\mathrm{TM}}$ LUX). J5 constitutive promoter [43] controlled eGFP expression, and the ribosome binding site (RBS site) was designed by RBS Calculator [44].

The dCas9 repression system consists of pCAS-ZE and pSEVA-eGFP. pCAS-ZE was derived from pCAS-RK2KdCas9. For construction of pCAS-ZE, kanamycin marker was replaced with tetracycline marker, and the $\lambda$-Red inducible expression system was eliminated from the backbone, and the N20 sequence was replaced with new sequence, targeting J5 promoter or RBS sites of eGFP. pSEVA-eGFP was assembled by connecting eGFP expression cassette with the AvrI/EcoRI digested pSEVA644. In the eGFP expression element, a ribosome RNA binding site $\left(5^{\prime}\right.$-GCGAGCGCGATCATTCTATTAGGGAGG GAGGT-3') was located between J5 promoter and fluorescent protein eGFP. According to CasOT, the original N20 sequence of pCAS-ZE was replaced with three new sites (by primers ZEJ-15F/ZEJ-15R, ZEJ-30F/ZEJ-30R and $Z E J-102 F / Z E J-102 R$ respectively), and these plasmids were named as pCAS-ZE1, pCAS-ZE2, pCAS-ZE3 respectively. Another N20 sequence (TGGATCGACCTT CGTACGAG) was cloned into pCAS-ZE0 via primers ZE-J5F/ZE-J5R, which was used as the positive control. Pseudomonas putida KT2440 was selected as the negative control.

After transformation of these plasmids into $P$. putida KT2440 as the protocol described above, the candidate strain was screened in tetracycline and gentamycin plates and identified by the colony PCR. Next, these strain were incubated in LB medium with appropriate antibiotics, and added $10 \mathrm{mM}$ rhamnose for the induction expression of gRNA cassette. Then related cells were harvested until $\mathrm{OD}_{600}$ reached $0.6-0.8$. Using the eGFP excitation wavelength at $480 \mathrm{~nm}$ and fluorescence emission at $510 \mathrm{~nm}$, a $200 \mu \mathrm{L}$ of undiluted cultivated cells was added into the 96-well microtiter plates and measured the absolute fluorescence intensity (AFI) and cell density $\left(\mathrm{OD}_{600}\right)$ by microplate reader. Finally, the fluorescence repression effect was reflected from the relative fluorescence intensity (RFI).

\section{Genome editing by CRISPR-Cpf1 system}

To generate CRISPR-Cpf1 system, firstly, the codon optimized FnCpf1 gene (from Francisella novicida) was cloned from pJYS1Ptac [45] (offered by Yang Sheng) by primers $\mathrm{pCf}-1 \mathrm{~F} / \mathrm{pCf}-2 \mathrm{R}$, and then $\mathrm{Cpf} 1$ fragment was cloned into pCAS-RK2K $\triangle$ Cas9 by Gibson assembly via primers $\mathrm{pCf}-2 \mathrm{~F} / \mathrm{pCf}-3 \mathrm{R}$ and $\mathrm{pCf}-3 \mathrm{~F} / \mathrm{pCf}-1 \mathrm{R}$, resulting in $\mathrm{pCpf1-RK2K}$. The 23 nt DNA sequence 'AGG CGCAGGGCCGCTTCTTTGAG' in pRO1600 replicon (pSEVA-gRNAT derivatives) was selected as target-curing site, which was assembled into pCpf1-RK2K (controlled by rhamnose-inducible promoter). In this FnCpf1 genome editing system, PP_3361 and PP_5301 were selected as target sites. Although FnCpf1 ( $5^{\prime}$ end 'TTN') and SpCas9 ( $3^{\prime}$ end 'NGG') recognize different PAM sequence, the repairing template can be the same donor DNA. Based on the backbone of pSEVA-gR3361T and pSEVA-gR5301T, we eliminated original Cas9-sgRNA sequence and added candidate Cpf1-crRNA into these plasmids via inverse PCR. To this end, pSEVA-gcR3361T 
and pSEVA-gcR5301T were constructed using primers 361-Cpf1F/361-Cpf1R and 5301-Cpf1F/5301-Cpf1R.

In this CRISPR-Cpf1 genome editing system, pCpf1RK2K was first transformed into $P$. putida KT2440 by conjugal transfer. After KT2440 harboring pCpf1-RK2K, crRNA plasmids were transformed into this strain by electroporation. pSEVA-gR3361T or pSEVA-gR5301T was selected as a control, which was introduced into KT2440 with an equal amount of DNA (150 ng). The related electroporation conditions, genome editing process, and plasmid curing method were consistent with above CRISPR-Cas9 system.

\section{Results}

\section{Establishment of a two-plasmid CRISPR-Cas9 system in $P$.} putida KT2440

Various CRISPR/Cas9 systems have been established in several common microorganisms. Taking the CRISPR/ Cas9 system in E. coli as example, it can be divided into a one-plasmid system [46], a two-plasmid system [29, 47] and a three-plasmid system [48]. Considering plasmid construction and the need for multiple genome editing rounds, we developed our system as a two-plasmid system. The E. coli CRISPR/Cas9 version 2.0 system developed by Jiang (unpublished) is a typical two-plasmid system that consists of several common inducible expression systems (e.g. araBAD expression system and rhaBAD expression system) and antibiotics markers used in Gram-negative bacteria. This system has also been extended into Tatumella citrea DSM 13699 [29]. Since Pseudomonas and E. coli are both $\gamma$-proteobacteria, many promoters and antibiotics markers share similar functions in these species. We therefore endeavored to develop a $P$. putida CRISPR/Cas9 two-plasmid system from Jiang's E. coli CRISPR/Cas9 version 2.0 system.

Firstly, we replaced the replicon of pCASsac with a broad-host-range replicon (pBBR1 or RK2), creating pCAS-pBBR1 and pCAS-RK2K (Fig. 1a), respectively. To prevent sucrose to be catalyzed by $\mathrm{SacB}$, we prepared electrocompetent cells with 3 mM HEPES buffer [13] instead of $300 \mathrm{mM}$ sucrose [49]. In the first step, we endeavored to introduce plasmid pCAS-RK2K or pCASpBBR1 into KT2440. After electroporation, we could not obtain a pCAS-pBBR1 transformant. Although we identified $P$. putida KT2440 colonies harboring pCASRK2K, the electroporation efficiency of this plasmid was low. Toxicity of Cas9 has been reported in several bacteria $[30,45,50]$, it was essential to understand whether the low transformation efficiency was caused by Cas9 toxicity, or low electroporation efficiency. Using the same electroporation parameters and an equal amount of DNA (150 ng), a series of Cas9 versions (pCAS-RK2KnCas9D, pCAS-RK2K-nCas9H, pCAS-RK2K-dCas9, and
pCAS-RK2K-Cas9FM) in pCAS-RK2K were respectively transformed into P. putida KT2440. As we know, different Cas9 mutation versions perform different cleavage effect or cannot cut DNA strand. In this study, different Cas9 versions made little difference to the numbers of the colony-forming units (CFU) obtained (Additional file 6). Therefore, we assumed that the low transformation efficiency obtained in the pCAS-RK2K electroporation experiments was due to low electroporation efficiency, not the lethality of Cas9. In the alternate approach, for pCAS-RK2K and its derivative plasmids, we could obtain a higher transformation efficiency by conjugation. Because the AadA proteins in Pseudomonads can degrade spectinomycin, we could use spectinomycin for the selection of P. putida KT2440 transconjugants [51, 52].

After generating P. putida KT2440 harboring pCASRK2K, we investigated whether Cas9 could be guided by sgRNA. We selected a non-essential gene (nicC) as the target site, and created pSEVA-gRicF and pSEVAdgRNA. As Fig. 2a shows, almost no cells survive RNA-guided cleavage of the Pseudomonas genome (pSEVA-gRicF plate), but some colonies grew when Cas9 was guided by an untargeted sequence (pSEVA-dgRNA plate). However, after addition of homologous repairing arm in pSEVA-gRicT, almost no colonies could survive on the selection plates. From these results, we ascribed cell death to the off-target effects of sgRNA. Calculating the similarity between the KT2440 genome and N20 sequences of pSEVA-gRicT via CasOT, we observed that the N20 sequence was not specifically targeted to the nicC gene, and there were similar sequences at different locations in the genome (Additional file 7). A specific N20 sequence was designed by CasOT [42] and inserted into pSEVA-gRicT to generate pSEVA-gRic6T (N20 sequence targeted to DNA template strand). After transformation, we obtained dozens of colonies (Fig. 2a, pSEVA-gRic6T plate) and the mutation rate was $100 \%$ in 10 randomly picked strains (Fig. 2c, d). Thus, this result indicated that target gene could be mutated via our CRISPR-Cas9 system.

To understand the relevance of four componentsCas9, the gRNA cassette, the homologous repairing arm, and $\lambda$-Red system-in our CRISPR/Cas9 system, we systematically constructed and transformed a series of plasmids into six groups to assess the connections among and requirements for these elements. Based on the research above, nic C was selected as the target site in this experiment. Based on total numbers of CFUs (Fig. 3), when Cas9 alone was introduced with pSEVA644 (Fig. 3a-I) or a non-target gRNA (Fig. 3a-III), we obtained a large number of transformants. Testing an otherwise intact CRISPR/Cas9 system without Cas9 protein (Fig. 3a-VI), the number of colonies was slightly higher than for the 


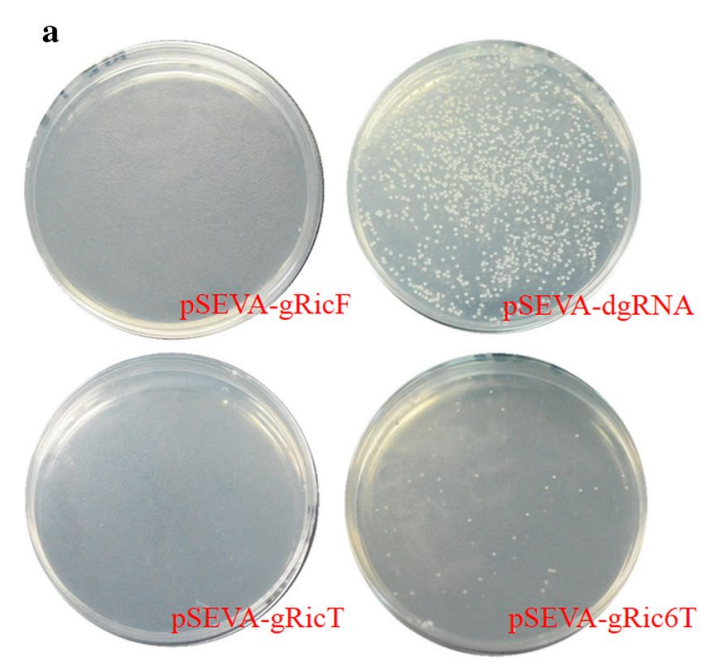

b

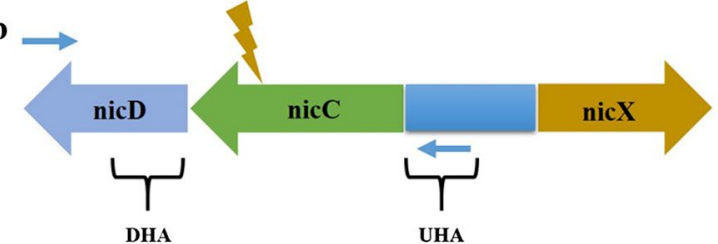

nicC (PP 3944)

c

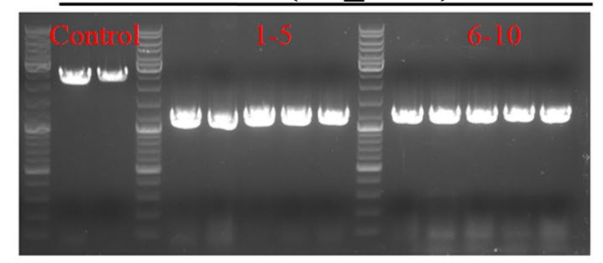

d

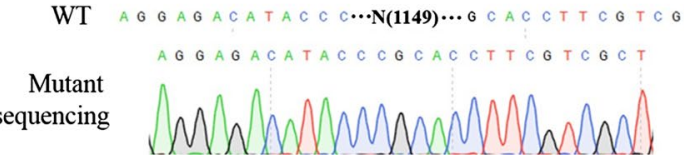

Fig. 2 CRISPR-Cas9 mediated nicC gene deletion in the Pseudomonas putida KT2440. a The phenotypes of pSEVA-gRNAT derivatives transformed into KT2440 harboring PCAS-RK2K. All plasmids were electrotransformed into pCAS-RK2K cells with an equal amount of DNA. b The schematic represents the design of identification primers for nic C gene deletion. Yellow arrow means the location of N2O sequence in nic $\mathrm{Cgene}$. Blue arrow represents the location of identification primers NT-JF and NT-JR. c Agarose gel electrophoresis shows the result of colony PCR to confirm nicC gene editing efficiency. $\mathbf{d}$ DNA sequencing proves that the 1149-nt nicC gene have been successfully deleted

two previous groups, indicating that the presence of Cas9 may exert slight cell toxicity or decrease the electroporation efficiency of small plasmids. Next, without the heterologous repairing ability provided by the $\lambda$-Red system (Fig. 3a-II, IV), we found cells hardly survived the Cas9induced DSBs using their endogenous repairing system, even on addition of a homologous repairing template (Fig. 3a-IV). In conclusion, only when the CRISPR/Cas9 system contains its four essential components (Fig. 3a-V) could efficient genome editing be achieved.

\section{Analysis of multigene editing efficiencies}

To further investigate the utility of CRISPR/Cas9 system in $P$. putida KT2440, we performed different genomic modification approaches in nicC gene site (Table 1). Firstly, it is essential to know the effect of N20 sequence targeting different DNA template strand. We designed pSEVA-gRic5T, which was a plasmid with a new N20 sequence targeting the DNA non-template strand. After electroporation of pSEVA-gRic5T, we picked 15 colonies from plates and found that the mutation rate reached 93.3\%. Thus, for the same target gene, a similar high mutation rate was observed when either DNA strand was targeted. Meanwhile, we also examined gene replacement of DNA fragment of three different lengths of (Table 1). All these fragments were respectively inserted between the upstream and downstream homologous arm of
pSEVA-gRic6T, and transformed into P. putida KT2440 harboring pCAS-RK2K using genome editing protocols described above. These insertions all showed a high knock-in rate (Table 1), which indicated that the length of the inserted fragment (within the range we tested) did not decrease the mutation rate.

In addition, we achieved single nucleotide mutation (SNM) in nicC gene, by changing the PAM sequence 'CGG' to 'CAG' via pSEVA-gRic6PAM2 (Table 1). Nevertheless, except for the PAM position, the editing of PAM unavailability sites was not so simple [53]. In order to achieve single nucleotide mutation for PAM unavailability sites, we need to select an ideal N20 sequence which can satisfy both non-off-target in genome and silent mutation (avoid repairing template degradation) will not change amino acid sequence. Next, we attempted to modify the Gln139 in nicC gene to Leu139 by mutating the codon 'CAA' to 'CTA'. In this experiment, we established a scarless two-step replacement strategy for SNM (Additional file 5), thus making a silent mutation in repairing template was not required. An A20 sequence was designed to insert between the N20 sequence and the PAM motif in pSEVA-gRic6PAM1, and this operation could result in replacement of the PAM motif of the original $\mathrm{N} 20$ sequence with the A20 sequence. In the first-step editing process, the target nucleotide was mutated and the A20 sequence was inserted into 

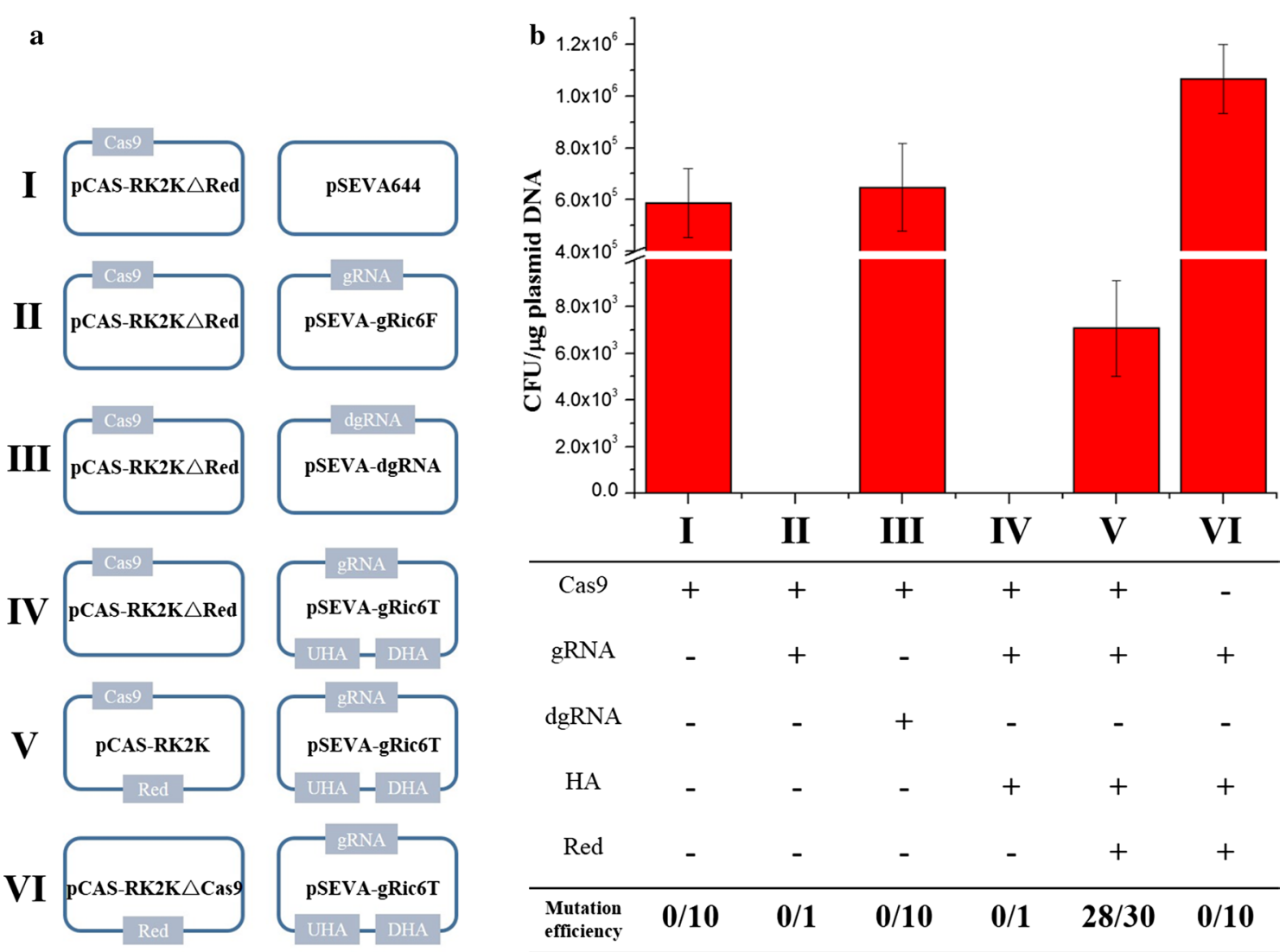

Fig. 3 Schematic diagram of the essential effects among four components (Cas9 protein, gRNA cassette, homologous arms and $\lambda$-Red system) in the Pseudomonas putida KT2440 genome editing. a The strategy of plasmids with different components were transformed into KT2440. pCAS-RK2K or its derivatives was first transformed into KT2440. In the second round of electroporation, pSEVA644, pSEVA-gRic6T and its derivatives were transformed into KT2440 harboring pCAS-RK2K relevant plasmids. b Electroporation efficiency is reflected from the total number of CFU (colony-forming units); Mutation efficiency in six groups with different components. The CFU experiment is obtained from three replicates. Cells were plated on the equal concentration antibiotics plates and the amount of DNA was equivalent in each experiment

the genome (Fig. 4b). Next, after curing of the pSEVANicA20, the A20 sequence was eliminated from the genome by transformation with pSEVA-NicA21. Finally, the single nucleotide mutation was retained in the target site (Fig. 4c).

Aside from these versatile mutations of nic $\mathrm{C}$ gene, nine other target sites, PP_0552, PP_3361, PP_3733, PP_3889, PP_3939-PP_3940, PP_3947-PP_3948, PP_1706, PP_3846 and PP_5301, were used to further examine the mutation rate of the CRISPR/Cas9 system. PP_0552, PP_3361, PP_3733 and PP_3889 were selected as genome editing sites in KT2440 harboring pCAS-RK2K. After transformation of pSEVA-gRNAT derivative plasmids (pSEVA-gR0552T, pSEVA-gR3361T, pSEVA-gR3733T and pSEVA-gR3889T) into KT2440 harboring pCAS-RK2K, we obtained high mutation rates in every case. The resulting mutation efficiencies for PP_0552, PP_3361, PP_3733 and PP_3889 were 80\% (8/10), 84.6\% (11/13), 100\% (18/18) and 91.6\% (11/12), respectively. Having demonstrated the mutation efficiency of KT2440 harboring pCAS-RK2K, the utility of pCAS-RK2T (tetracycline version) was examined in another five sites (PP_3947-PP_3948, PP_3939-PP_3940, PP_3846, PP_1706 and PP_5301). After electroporation of pSEVA-gR3947-3948T into KT2440 harboring pCASRK2T, all eighteen mutant clones were proved to be successful editing. In the case of PP3939-PP3940, nine out of ten transformants were mutated. When pSEVA-gR3846T was introduced into KT2440 containing pCAS-RK2T, the editing efficiency of PP_3846 was 100\% (10/10), similar to the frequency (18/18) obtained from PP_3947-PP_3948 mutation experiment. For PP_1706 and PP_5301, the colony PCR indicated that mutation efficiencies of $93.7 \%$ $(15 / 16)$ and $100 \%(10 / 10)$ were generated in relevant experiment. Comparison of the deletion rate at the nine different locations is given in Table 1 . The mutation rate varied between sites, but the editing efficiency overall was high. 
Table 1 Mutation efficiency of the type II CRISPR system in P. putida KT2440

\begin{tabular}{|c|c|c|c|c|c|c|c|}
\hline Host cell & gRNA plasmid & Deletion & Replacement & Insertion & Size & Results & $\begin{array}{l}\text { Plasmid } \\
\text { curing } \\
\text { efficiency }\end{array}$ \\
\hline KT2440 harboring pCAS-RK2K & pSEVA-gRic6T & nicC (PP_3944) & & & 1149 & $15 / 15$ & ND \\
\hline KT2440 harboring pCAS-RK2K & pSEVA-gRic5T & nicC (PP_3944) & & & 1149 & $39 / 45$ & ND \\
\hline KT2440 harboring pCAS-RK2K & pSEVA-gRic6T $\triangle$ NicC::rhla & nicC (PP_3944) & rhla & & 888 & $38 / 40$ & ND \\
\hline KT2440 harboring pCAS-RK2K & pSEVA-gRic6T $\triangle N i c C:: d$ Cas9 & nicC (PP_3944) & dCas 9 & & 4107 & $20 / 20$ & ND \\
\hline KT2440 harboring pCAS-RK2K & $\begin{array}{l}\text { pSEVA-gRic6T } \triangle N i c C:: T 7 \text { RNA } \\
\text { polymerase }\end{array}$ & nicC (PP_3944) & $\mathrm{T7}$ & & 4518 & $14 / 20$ & $5 / 5$ \\
\hline KT2440 harboring pCAS-RK2K & pSEVA-gRic6PAM2 & Single nucleotide & Single nucleotide & & 1 & $5 / 5$ & ND \\
\hline KT2440 harboring pCAS-RK2K & pSEVA-NicA20 & Single nucleotide & Single nucleotide & $\mathrm{A} 20$ & 21 & $7 / 7$ & ND \\
\hline KT2440 harboring pCAS-RK2K & pSEVA-NicA21 & $\mathrm{A} 20$ & & & 20 & $5 / 5$ & ND \\
\hline KT2440 harboring pCAS-RK2K & pSEVA-gR0552T & PP_0552 & & & 1089 & $8 / 10$ & ND \\
\hline KT2440 harboring pCAS-RK2K & pSEVA-gR3361T & PP_3361 & & & 3048 & $11 / 13$ & ND \\
\hline KT2440 harboring pCAS-RK2K & pSEVA-gR3733T & PP_3733 & & & 1059 & 18/18 & ND \\
\hline KT2440 harboring pCAS-RK2K & pSEVA-gR3889T & PP_3889 & & & 1299 & $11 / 12$ & ND \\
\hline KT2440 harboring pCAS-RK2T & pSEVA-gR3947-3948T & PP_3947-PP_3948 & & & 4033 & $18 / 18$ & $5 / 5$ \\
\hline KT2440 harboring pCAS-RK2T & pSEVA-gR3939-3940T & PP_3939-PP_3940 & & & 2743 & $9 / 10$ & $5 / 5$ \\
\hline KT2440 harboring pCAS-RK2T & pSEVA-gR3846T & PP_3846 & & & 816 & $10 / 10$ & ND \\
\hline KT2440 harboring pCAS-RK2T & pSEVA-gR1706T & PP_1706 & & & 363 & $15 / 16$ & ND \\
\hline KT2440 harboring pCAS-RK2T & pSEVA-gR5301T & PP_5301 & & & 264 & $10 / 10$ & ND \\
\hline KT2440 harboring pCpf1-RK2K & pSEVA-gcR3361T & PP_3361 & & & 3048 & $4 / 4$ & ND \\
\hline KT2440 harboring pCpf1-RK2K & pSEVA-gcR5301T & PP_5301 & & & 264 & $9 / 9$ & $2 / 2$ \\
\hline
\end{tabular}

rhla was cloned from Pseudomonas aeruginosa PAOI, and T7 RNA polymerase was amplified from Escherichia coli BL21(DE3)

$N D$ not determined

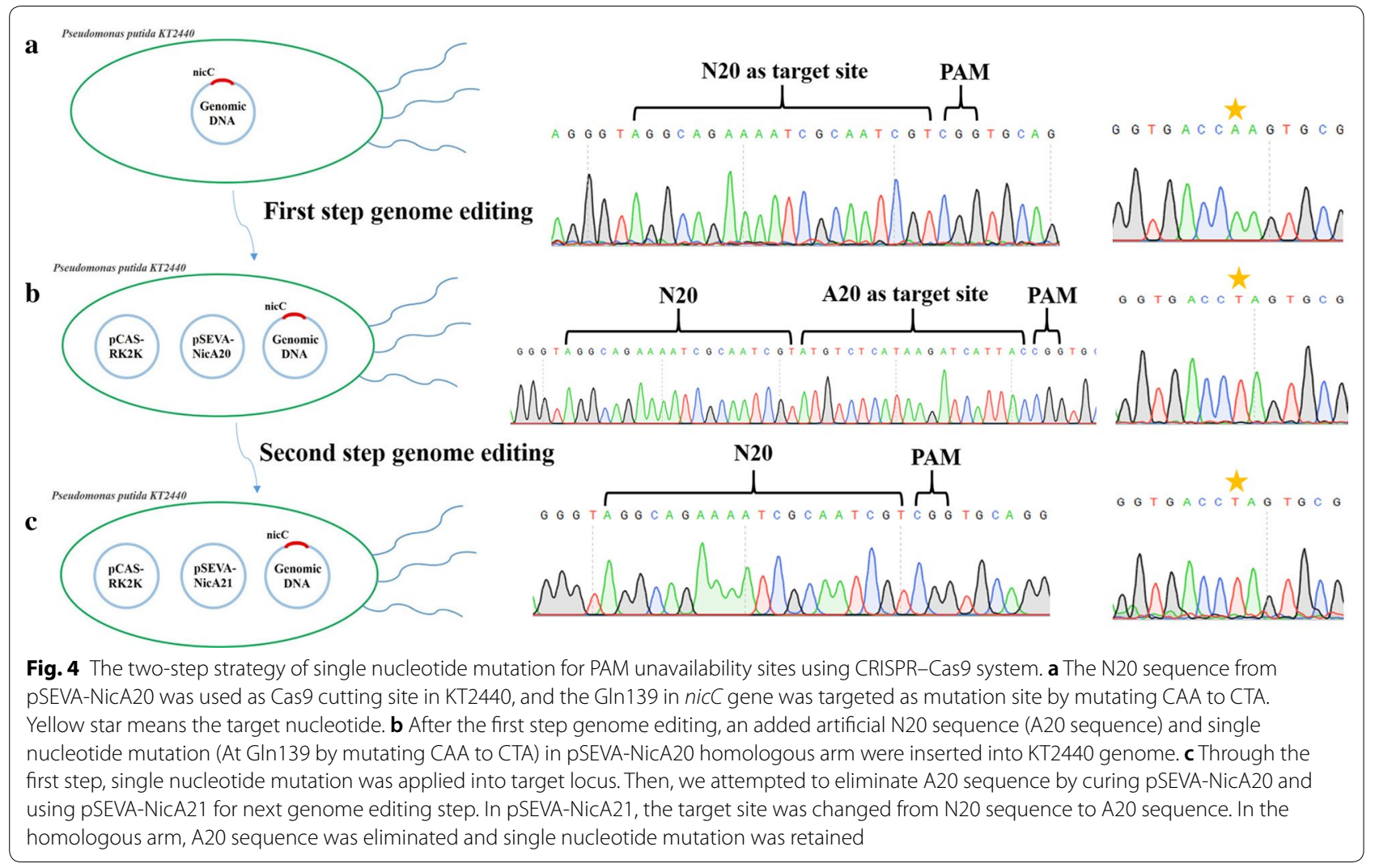




\section{Interference the expression of eGFP by dCas 9}

To expand the function of our CRISPR/Cas9 system, we endeavored to establish a CRISPR interference (CRISPRi) system in P. putida KT2440. In CRISPRi, a nuclease deficient Cas9 (dCas9) can be guided by a sgRNA or multi sgRNAs to exert transcriptional repression upstream of target genes. Without its endonucleolytic activity, dCas9 remains bound to the target locus; this has been validated for regulation of gene expression in several organisms $[27,28,50]$.

We attempted to demonstrate a CRISRPi effect via the expression intensity of enhanced green fluorescent protein (eGFP) in pSEVA-J5-eGFP (Fig. 5a). Three sgRNAs targeted to different loci of eGFP transcription were designed and inserted into pCAS-ZE1, pCAS-ZE2, and pCAS-ZE3 respectively (Fig. 5b). After two-step electrotransformation or co-electrotransformation, KT2440 harboring dCas9 and
eGFP was screened and incubated in LB medium containing antibiotics and rhamnose. After overnight cultivation, the cell concentration $\left(\mathrm{OD}_{600 \mathrm{~nm}}\right)$ and the absolute fluorescence intensity (AFI) were measured simultaneously in a microplate reader. The final fluorescence repression results were evaluated in terms of relative fluorescence intensity (RFI) (Fig. 5d), which was calculated as AFI divided by $\mathrm{OD}_{600}$. Compared to the pCAS-ZE0 control group (N20 sequence lacked target site), approximately $350 \%$ and $340 \%$ fluorescence repression effects were obtained in groups ZE1 (N20 sequence targeted to the template strand) and ZE2 (N20 sequence targeted to the nontemplate strand) when the sgRNA associated with dCas9 binding to the - 35 position in the J5 promoter, on either DNA strand. Only a slight repression effect (138\%) was achieved in the group ZE3 (N20 sequence targeted to RBS sites on the nontemplate strand). As for groups ZE1 and ZE2,

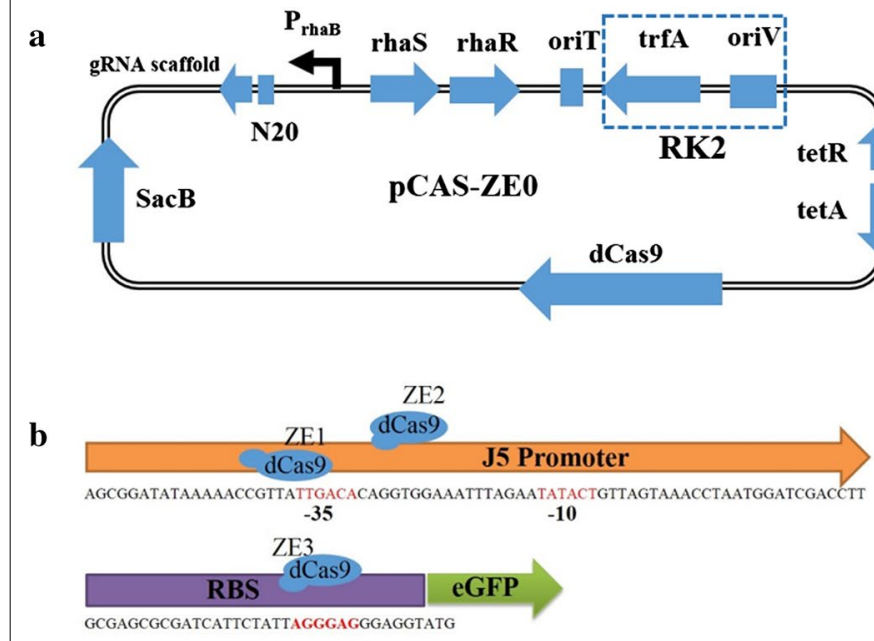

c
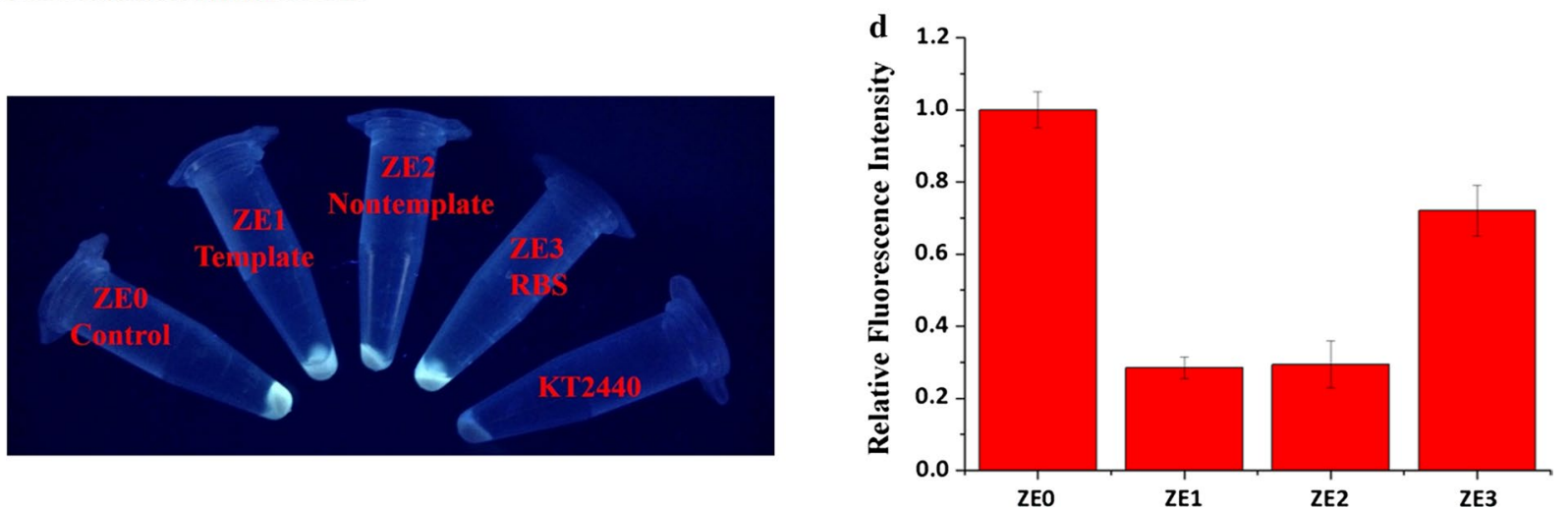

Fig. 5 CRISPR-dCas9 mediated transcription inhibition in the Pseudomonas putida KT2440. a Schematic representation of pCAS-ZEO and its derivatives (pCAS-ZE1, pCAS-ZE2, pCAS-ZE3) used for transcription inhibition. Plasmid-borne enhanced green fluorescence protein (eGFP) was selected as target site. $\mathbf{b}$ Illustration of different dCas9 binding sites are indicated in the upstream sequence of plasmid pSEVA-eGFP. ZE1 and ZE2 were targeting the -35 region of 55 promoter, and ZE3 was binding with Ribosome Binding Site (RBS). To examine the effect of selecting different DNA strand, ZE1 was designed to bind with template strand and ZE2 was located at the non-template strand. c KT2440 cells harboring dCas9 and eGFP plasmids were gathered with equal amount and exposed under UV light. Blank KT2440 cells were used as control. d Comparsion of the repression effectiveness of dCas9 binding with different target sites 
similar repression effects were achieved by targeting different DNA template strands when dCas9 targeted to - 35 region in the promoter. This repression efficiency indicated that dCas9 exhibited no strand bias to -35 region in the promoter. In case of group ZE3, the repression activity of dCas9 was significantly decreased compared to groups ZE1 and ZE2. These results demonstrated that a different dCas9-binding site (promoters or RBS sites) led to distinct repression of intensity. Because of the obvious fluorescence of eGFP, we could also observe transcriptional blocking by the naked eyes. Samples from each group were concentrated in respective $1.5-\mathrm{mL}$ Eppendorf tubes and exposed to UV light. The results (Fig. 5c) were fully consistent with the RFI parameters. Altogether, these data demonstrate that dCas9 could be an efficient tool for gene repression in P. putida KT2440.

\section{Application of CRISPR-Cpf1 genome editing system in $P$. putida KT2440}

We also endeavored to explore the feasibility of using FnCpf1 for genome editing in P. putida KT2440. We found that pCpf1-RK2K could only be transferred into KT2440 by conjugal transfer. Although we obtained dozens of clones by electroporation, pCpf1-RK2K could not be identified in these cells.
After preparation of electrocompetent KT2440 harboring pCpf1-RK2K, plasmids pSEVA-gcR3361T, pSEVAgcR5301T and control plasmids (pSEVA-gR3361T and pSEVA-gR5301T) were transformed by electroporation, respectively. Using the same procedure as for Cas9 genome editing, several random cells were picked from plates and tested by colony PCR. Using this CRISPRCpf1 system, we achieved deletion rate of $100 \%$ in PP_3361 (Fig. 6b), and PP_5301 (Fig. 6b). Thus, we preliminarily demonstrated that FnCpf1 can be harnessed as a genome editing tool in P. putida KT2440.

\section{Discussion}

In this study, we have demonstrated that a CRISPR/ Cas9 system can be used for genome editing and regulation of gene expression in P. putida KT2440. A two-plasmid CRISPR/Cas9 system [29] was selected as our backbone because of the need for continual genome editing. In contrast to previous genome editing approaches [5-17] and a recently reported CRISPRCas9 method [37] (Table 2), our CRISPR/Cas9 system prove to be an efficient and fast tool in $P$. putida KT2440. The CRISPR/Cas9 genome editing system in P. putida is first developed by Aparicio et al. [37], in which gene deletion is performed by Cas 9 protein

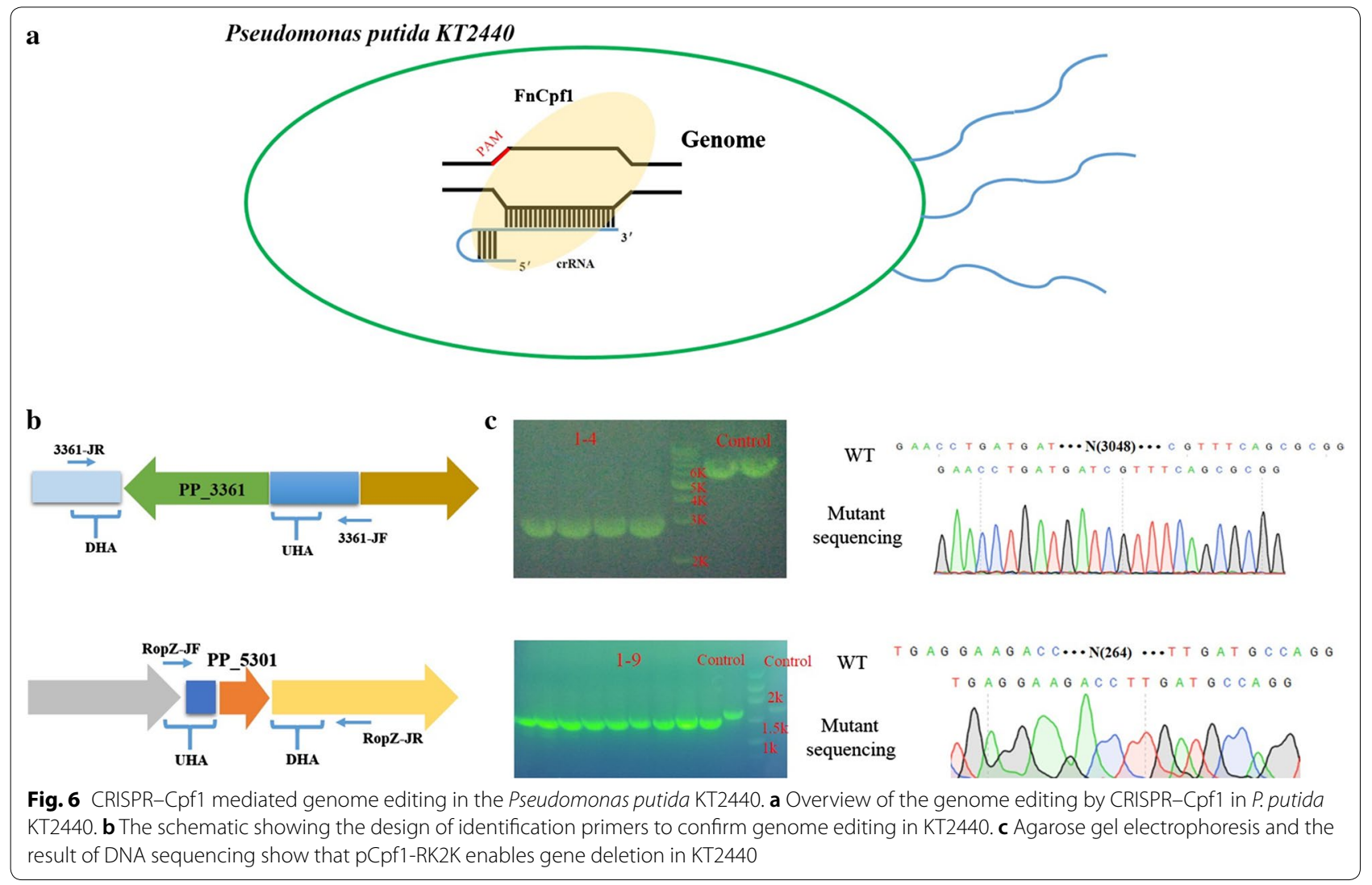




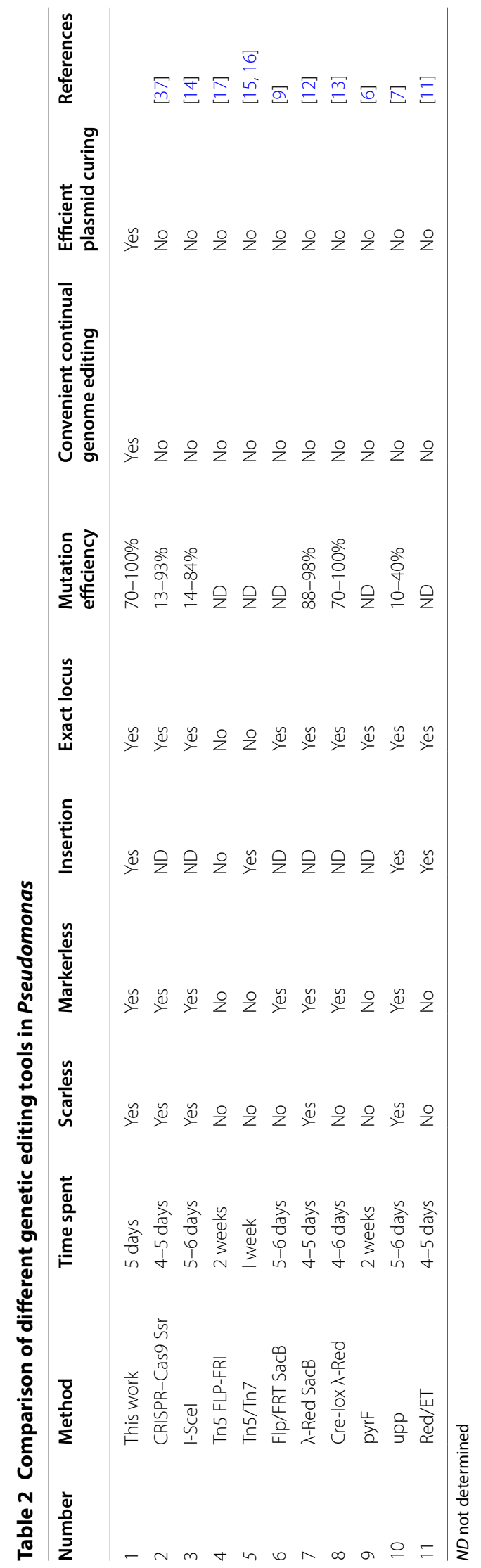


Day 1 6:00 a.m. Electroporation

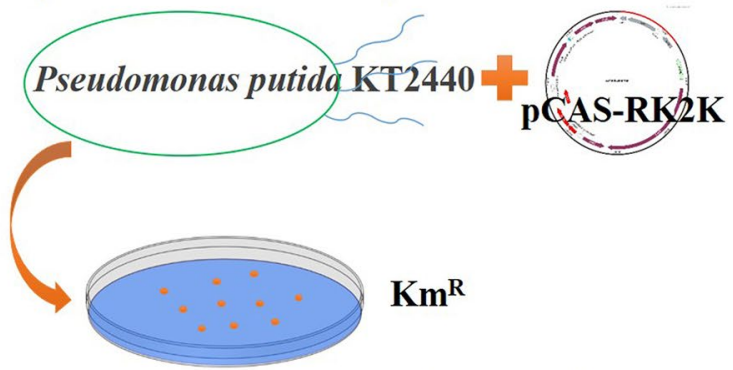

Cure pSEVA-gRNAT

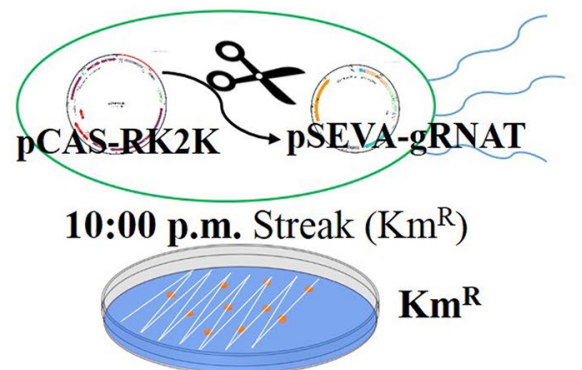

10:00 p.m.-11:00 p.m. Inoculation $\left(\mathrm{Km}^{\mathrm{R}}\right) \quad$ Day 4 10:00 a.m. Colony PCR, inoculation $\left(\mathrm{Km}^{\mathrm{R}}\right)$

Day 2 10:00 a.m. - 2:00 p.m. Cultivation, induction and electroporation
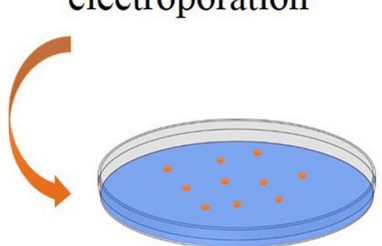

$\mathbf{K m}^{\mathrm{R}}+\mathbf{G m}^{\mathrm{R}}$

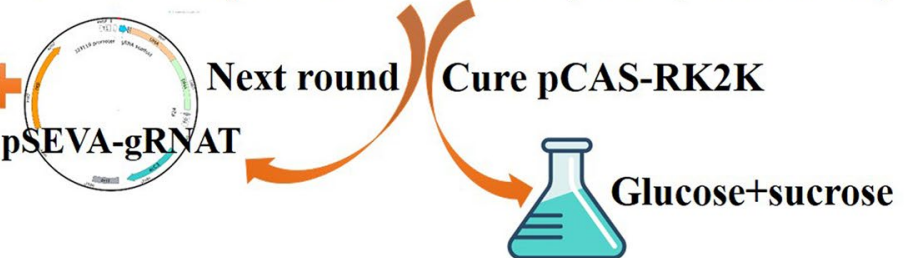

10:00 p.m.-11:00 p.m. Streak
Day 3 8:00 a.m. Colony PCR,
inoculation $\left(\mathrm{Km}^{\mathrm{R}}+\right.$ rhamnose $)$
Glucose+sucrose

Fig. 7 Diagram for the CRIPSR-Cas9-assisted genome editing in P. putida KT2440. Day 1: Introduce the pCAS-RK2K plasmid into P. putida KT2440, and then inoculate the transformants in LB medium overnight; Day 2: Transfer the cultivated cells into fresh LB medium and add arabinose to trigger expression of $\lambda$-Red proteins. Next, cells were prepared as elecompetent cells and the pSEVA-gRNAT plasmids were transferred to KT2440 harboring PCAS-RK2K. Day 3: Screen out the mutants by colony PCR. The mutants were inoculated in LB medium containing antibiotic and rhamnose in the morning; Streak the cultivated cells on LB agar in the evening; Day 4: Screen out the mutants that have lost the pSEVA-gRNAT plasmids, and inoculate the mutants in LB medium containing glucose and sucrose. Next, in the evening, the cultivated cells were streak on plate containing glucose and sucrose, and then cultivated overnight. The mutants that have been cured of pSEVA-gRNAT can be used for the next round of genome editing; Day 5: Identify the mutants from the selection plate

assisting with single strand DNA (ssDNA) and a recombinase protein (Ssr) in a three-plasmid system. However, the genome editing efficiency in this approach has highly variable efficiency (13-93.2\% below $5 \mathrm{~kb}$ target), gene insertion and replacement cannot be realized, and a plasmid curing strategy is also suboptimal. In our CRISPR/Cas9 system, genome editing efficiency reached more than $70 \%$, and the total process including gene editing and plasmid curing could be achieved within 5 days (Fig. 7), which outcompetes most previous approaches in Pseudomonas. Owing to the high efficient plasmid-curing strategy, for continual genome editing, we can perform three rounds of genome editing in 1 week (Additional file 8). Besides gene deletion and insertion, transcriptional repression caused by dCas9 via different expression intensities of eGFP was successfully demonstrated in our study. In addition, we established another type II CRISPR system in KT2440, the CRISPR-Cpf1 system, which showed high genome editing efficiency.
To establish a new genome editing approach in $P$. putida KT2440 via the CRISPR-Cas9 system, we first considered the transformation method for our plasmids. In our study, we applied two broad-host-range replicons for pCASsac function in Pseudomonas: pCAS-pBBR1 could not be transformed into hosts via electroporation, and the electroporation efficiency of pCAS-RK2K was low. Due to previous reports of Cas 9 toxicity in several bacteria [30, 45, 50], we constructed five Cas9 protein versions that were transformed into $P$. putida KT2440 for toxicity analysis. There was no obvious difference of transformation efficiency between the five Cas9 versions, thus we could eliminate Cas9 toxicity as a major issue.

In the next sgRNA construction step, we demonstrated Cas 9 protein could be guided by sgRNA, which killed almost all the cells in the nic $C$ plates. In the following procedures, however, we could hardly obtain any transformants from screening plates even with addition of donor DNA and the help of heterologous recombination proteins. Obviously, off-target effect of Cas9 
was another challenge in this study, and could cause Cas9 to target other locations in the genome. Without a homologous repairing template, Pseudomonas cells could hardly survive multiple genome breaks. By means of CasOT, we could design sequence to avoid cells being cut by Cas 9 at off-target sites. Finally (Fig. 2), transformants screened from plates showed a high knock-out efficiency. Due to the relative lower frequencies of native homology-directed repair system in P. putida KT2440, $\lambda$-Red-mediated recombination was essential for genome editing to proceed (Fig. 3). Additionally, we tried to simplify the construction of pSEVA-gRNAT plasmids, e.g. the homologous repairing arm supplied as a fragment was co-transformed with pSEVA-gRNAF (lacked the homologous repairing arm) into KT2440 harboring pCAS-RK2K. Although we could obtain dozens of transformants on plates, the mutation rate of target site dramatically decreased to $10 \%$. In previous other genome editing methods [14], we found donor DNA was inserted into plasmids, and we assumed this phenomenon might be correlated with a lower efficiency of DNA uptake in $P$. putida KT2440.

After the preliminary establishment of the CRISPR/ Cas9 system, we attempted different genome editing approaches in P. putida KT2440. We showed that an N20 sequence could obtain a similar high mutation rate irrespective of the target DNA template strand, and the efficiency of fragment insertion was not affected by the length of the fragment (within the range we tested). In addition, we developed a single nucleotide mutation approach for different target site conditions. A Onestep method could be performed if the desired site is in a PAM motif or the last $12 \mathrm{bp}$ of the N20 sequence near a PAM region [53]. However, not every nucleotide satisfies these requirements, and for those we developed a two-step strategy. We designed a high-specific 20-bp sequence (A20 sequence) between the N20 sequence and PAM motif, which could avoid the degradation of donor DNA. After the first step, single nucleotide could be mutated and the A20 sequence left in the genome. Then, in the next step, we changed $\mathrm{N} 20$ sequence to this A20 site, and then A20 sequence was eliminated from homologous repairing template. By this method, we could obtain a scarless single nucleotide modified strain.

Cas9 can be guided by multi sgRNAs, and multi-gene editing performed by CRISPR/Cas9 is a unique feature compared with other genome editing tools. In our study, we also attempted to perform a double-locus editing experiment, but could not obtain a strain with double-locus deletion after we picked more than 100 transformants in one experiment. Although gene deletion simultaneously performed in two sites was successfully demonstrated in a recent study [37], the editing efficiency of this method was extremely low $(0.5 \%)$. In our study, we do not recommend this method because of its low efficiency and time-consuming plasmid construction. Instead, we tended to perform multigene editing by multiple rounds, achieving genomic mutation in a single target site in each round. This approach was highly efficient, and the manipulation time was reduced via the flexible and efficient curing strategy in our two-plasmid system.

CRISPR interference is an alternative approach to gene knock-out for regulation of gene expression and has been widely used in bacteria such as E. coli [54] and Clostridium [50]. Here, we selected eGFP and tested different dCas9 binding sites as target sites for CRISPRi. Different dCas9 target sites had a significant impact on the level of transcriptional repression, but the DNA strand on which an N20 sequence was located had no obvious effect on dCas9 blocking of gene expression. When dCas9 targeted to the starting region of promoter, this repression effect was much better than RBS sites. The exploration of pCAS-ZE systems could be a useful approach for metabolic engineering regulation and other genomic manipulation in P. putida KT2440.

The CRISPR-Cpf1 system is an emerging Type II CRISPR genome editing tool, which has also been established in several organisms $[45,55,56]$. Due to its longer target sequence (23-25 nt), Cpf1 exhibits relatively low potential for off-target activity, creating applicability of CRISPR-Cpf 1 as a complementary genome editing tool to CRISPR-Cas9 system [38]. The establishment in the study of the CRISPR-Cpf1 system in strain KT2440 will be an alternative tool for some gene loci, for which it is hard to obtain a non-off-target N20 sequence.

\section{Conclusions}

Here, high efficient and versatile functions CRISPR/ Cas9 system have been successfully established in $P$. putida KT2440. Although off-target effects of Cas9 are a challenge in target locus selection, versatile gRNA selection tools $[42,57,58]$ and an enhanced-specificity mutant version of Cas9 have been developed [59]; these tools can minimize off-target effects and increase ontarget possibilities. Besides P. putida, there are abundant Pseudomonas species, such as $P$. aeruginosa, a commonly used strain in immunology [60], and $P$. fluorescens, which possess growth-promoting ability for host plant [61]. Currently, we are trying to extend the CRISPR/Cas9 system and the CRISPR/Cpf1 system into $P$. aeruginosa PA01 and $P$. fluorescens $\mathrm{PfO}-1$ in our laboratory. $P$. putida KT2440 is a well-known "generally recognized as safe" (GRAS) bacterium, a versatile platform in biotechnology [62], and an important next 
generation synthesis biology chassis [63]. Although the complete genome of $P$. putida KT2440 was sequenced [64] and revisited [65], and a set of bioinformatics tools $[66,67]$ was also developed, the practical biotechnological methods for predictive verification and modification of these chassis cells are suboptimal. Thus, the establishment of CRISPR-Cas9 and CRISPR-Cpf1 systems in strain KT2440 will spur and facilitate further understanding and application of this strain, and form the basis to extend CRISPR genome systems into other important Pseudomonas strains.

\section{Additional files}

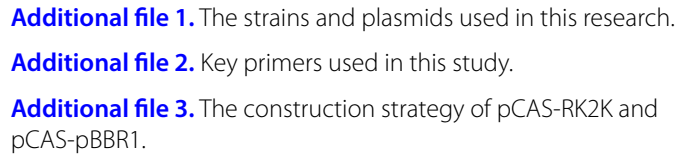

Additional file 4. PAM-guide sequences used in related plasmids.

Additional file 5. Plasmids of two-step single nucleotide mutation strategy.

Additional file 6. The total CFU calculation from different Cas9 versions.

Additional file 7. The analysis of sgRNA off-target in KT2440 by CasOT.

Additional file 8. Flow-chart of 3 rounds of continual genome editing in P. putida KT2440.

\begin{abstract}
Abbreviations
CFU: colony-forming units; CRISPRi: clustered regularly interspaced short palindromic repeat interference; crRNA: CRISPR RNA; DSB: double-stranded breaks; DHA: downstream homologous arm; dCas9: nuclease-deficient Cas9; eGFP: enhanced green fluorescent protein; GRAS: generally regarded as safe; LB: Luria-Bertani; PAM: protospacer-adjacent motif; RBS: ribosome binding site; SNM: single nucleotide mutation; sgRNA: single-guide RNA; UHA: upstream homologous arm.
\end{abstract}

\section{Authors' contributions}

$J W, S Y$ and JS designed the experiments. LY, YJ, QW, ZW and JS participated in the discussion about the experiments. JS performed experiments and drafted the manuscript. All authors read and approved the final manuscript.

\section{Author details}

${ }^{1}$ Institute of Bioengineering, College of Chemical and Biological Engineering, Zhejiang University, Hangzhou 310027, China. ${ }^{2}$ Key Laboratory of Synthetic Biology, Institute of Plant Physiology and Ecology, Shanghai Institutes for Biological Sciences, Chinese Academy of Sciences, Shanghai 200032, China. ${ }^{3}$ Shanghai Research and Development Center of Industrial Biotechnology, Shanghai 201206, China. ${ }^{4}$ Jiangsu National Synergetic Innovation Center for Advanced Materials, SICAM, Nanjing 210009, China.

\section{Acknowledgements}

We would like to thank Esteban Martínez-García for providing the pSEVA plasmids. We also thank Pei Lei (from Huazhong Agricultural University) for kind donation of P. putida KT2440 and Escherichia coli S17-1.

\section{Competing interests}

The authors declare that they have no competing interests.

\section{Availability of data and materials}

All supporting data is present in the article and the additional files. pCASRK2T, pSEVA-gRic6T and pCAS-ZE3 are available on Addgene (under the Numbers 106400, 106401 and 106402) and GenScript (under the Numbers MC 0000261, MC 0000262 and MC 0000263).
Consent for publication

Not applicable.

Ethics approval and consent to participate

Not applicable.

Funding

This work was financially supported by the National Natural Science Foundation of China (No. 21676240).

\section{Publisher's Note}

Springer Nature remains neutral with regard to jurisdictional claims in published maps and institutional affiliations.

Received: 8 November 2017 Accepted: 3 March 2018

Published online: 13 March 2018

\section{References}

1. Nikel PI, Martínez-García E, de Lorenzo V. Biotechnological domestication of pseudomonads using synthetic biology. Nat Rev Microbiol. 2014;12:368-79.

2. Gong T, Liu R, Zuo Z, Che Y, Yu H, Song C, et al. Metabolic engineering of Pseudomonas putida KT2440 for complete mineralization of methyl parathion and $y$-hexachlorocyclohexane. ACS Synth Biol. 2016;5:434-42.

3. Kuepper J, Dickler J, Biggel M, Behnken S, Jäger G, Wierckx N, et al. Metabolic engineering of Pseudomonas putida KT2440 to produce anthranilate from glucose. Front Microbiol. 2015;6:1-9.

4. Wang Y, LV M, Zhang Y, Xiao X, Jiang T, Zhang W, et al. Reconstruction of lactate utilization system in Pseudomonas putida KT2440: a novel biocatalyst for I-2-hydroxy-carboxylate production. Sci Rep. 2015;4:6939.

5. Hmelo LR, Borlee BR, Almblad H, Love ME, Randall TE, Tseng BS, et al. Precision-engineering the Pseudomonas aeruginosa genome with twostep allelic exchange. Nat Protoc. 2015;10:1820-41.

6. Galvão TC, De Lorenzo V. Adaptation of the yeast URA3 selection system to gram-negative bacteria and generation of a $\triangle$ betCDE Pseudomonas putida strain. Appl Environ Microbiol. 2005;71:883-92.

7. Graf N, Altenbuchner J. Development of a method for markerless gene deletion in Pseudomonas putida. Appl Environ Microbiol. 2011;77:5549-52.

8. Quénée L, Lamotte D, Polack B. Combined sacB-based negative selection and cre-lox antibiotic marker recycling for efficient gene deletion in Pseudomonas aeruginosa. Biotechniques. 2005;38:63-7.

9. Hoang TT, Karkhoff-schweizer RR, Kutchma AJ, Schweizer HP. A broadhost-range Flp-FRT recombination system for site-specific excision of chromosomally-located DNA sequences: application for isolation of unmarked Pseudomonas aeruginosa mutants. Gene. 1998;212:77-86.

10. Ortiz-Martín I, Macho AP, Lambersten L, Ramos C, Beuzón CR. Suicide vectors for antibiotic marker exchange and rapid generation of multiple knockout mutants by allelic exchange in Gram-negative bacteria. J Microbiol Methods. 2006;67:395-407.

11. Wenzel SC, Gross F, Zhang Y, Fu J, Stewart AF, Müller R. Heterologous expression of a myxobacterial natural products assembly line in pseudomonads via Red/ET recombineering. Chem Biol. 2005;12:349-56.

12. Liang R, Liu J. Scarless and sequential gene modification in Pseudomonas using PCR product flanked by short homology regions. BMC Microbiol. 2010;10:209.

13. Luo X, Yang Y, Ling W, Zhuang H, Li Q, Shang G. Pseudomonas putida KT2440 markerless gene deletion using a combination of $\lambda$ Red recombineering and Cre/loxP site-specific recombination. FEMS Microbiol Lett. 2016;363:1-7.

14. Martínez-García E, de Lorenzo V. Engineering multiple genomic deletions in Gram-negative bacteria: analysis of the multi-resistant antibiotic profile of Pseudomonas putida KT2440. Environ Microbiol. 2011;13:2702-16.

15. Zobel S, Benedetti I, Eisenbach L, De Lorenzo V, Wierckx N, Blank LM. Tn7-based device for calibrated heterologous gene expression in Pseudomonas putida. ACS Synth Biol. 2015;4:1341-51. 
16. Martínez-García E, Calles B, Arévalo-Rodríguez M, de Lorenzo V. pBAM1: an all-synthetic genetic tool for analysis and construction of complex bacterial phenotypes. BMC Microbiol. 2011;11:38.

17. Leprince A, de Lorenzo V, Völler P, van Passel MWJ, Martins dos Santos VAP. Random and cyclical deletion of large DNA segments in the genome of Pseudomonas putida. Environ Microbiol. 2012;14:1444-53.

18. Sapranauskas R, Gasiunas G, Fremaux C, Barrangou R, Horvath P, Siksnys V. The Streptococcus thermophilus CRISPR/Cas system provides immunity in Escherichia coli. Nucleic Acids Res. 2011:39:9275-82.

19. Makarova KS, Wolf Yl, Alkhnbashi OS, Costa F, Shah SA, Saunders SJ, et al. An updated evolutionary classification of CRISPR-Cas systems. Nat Rev Microbiol. 2015;13:722-36.

20. Jinek M, Chylinski K, Fonfara I, Hauer M, Doudna JA, Charpentier E. A programmable dual-RNA-guided DNA endonuclease in adaptive bacterial immunity. Science. 2012;337:816-21.

21. Mojica FJM, Díez-Villaseñor C, García-Martínez J, Almendros C. Short motif sequences determine the targets of the prokaryotic CRISPR defence system. Microbiology. 2009;155:733-40.

22. Gasiunas G, Barrangou R, Horvath P, Siksnys V. Cas9-crRNA ribonucleoprotein complex mediates specific DNA cleavage for adaptive immunity in bacteria. Proc Natl Acad Sci. 2012;109:E2579-86.

23. Sung $\mathrm{P}$, Klein $\mathrm{H}$. Mechanism of homologous recombination: mediators and helicases take on regulatory functions. Nat Rev Mol Cell Biol. 2006;7:739-50.

24. Guirouilh-Barbat J, Huck S, Bertrand P, Pirzio L, Desmaze C, Sabatier L, et al. Impact of the KU80 pathway on NHEJ-induced genome rearrangements in mammalian cells. Mol Cell. 2004;14:611-23.

25. Cong L, Ran F, Cox D, Lin S, Barretto R, Habib N, et al. Multiplex genome engineering using CRISPR/Cas systems. Science. 2013;339:819-22.

26. Metzger MJ, McConnell-Smith A, Stoddard BL, Miller AD. Single-strand nicks induce homologous recombination with less toxicity than double-strand breaks using an AAV vector template. Nucleic Acids Res. 2011:39:926-35.

27. Cress BF, Toparlak OD, Guleria S, Lebovich M, Stieglitz JT, Englaender JA, et al. CRISPathBrick: modular combinatorial assembly of type II-A CRISPR arrays for dCas9-mediated multiplex transcriptional repression in E. coli. ACS Synth Biol. 2015:4:987-1000

28. Bikard D, Jiang W, Samai P, Hochschild A, Zhang F, Marraffini LA. Programmable repression and activation of bacterial gene expression using an engineered CRISPR-Cas system. Nucleic Acids Res. 2013:41:7429-37.

29. Jiang Y, Chen B, Duan C, Sun B, Yang J, Yang S. Multigene editing in the Escherichia coli genome via the CRISPR-Cas9 system. Appl Environ Microbiol. 2015;81:2506-14.

30. XuT, Li Y, Shi Z, Hemme CL, Li Y, Zhu Y, et al. Efficient genome editing in Clostridium cellulolyticum via CRISPR-Cas9 nickase. Appl Environ Microbiol. 2015;81:4423-31.

31. Cobb RE, Wang Y, Zhao H. High-efficiency multiplex genome editing of Streptomyces species using an engineered CRISPR/Cas system. ACS Synth Biol. 2015:4:723-8.

32. Westbrook AW, Moo-Young M, Chou CP. Development of a CRISPR-Cas9 tool kit for comprehensive engineering of Bacillus subtilis. Appl Environ Microbiol. 2016;82:4876-95.

33. Gao S, Tong Y, Wen Z, Zhu L, Ge M, Chen D, et al. Multiplex gene editing of the Yarrowia lipolytica genome using the CRISPR-Cas9 system. J Ind Microbiol Biotechnol. 2016:43:1085-93.

34. Dicarlo JE, Norville JE, Mali P, Rios X, Aach J, Church GM. Genome engineering in Saccharomyces cerevisiae using CRISPR-Cas systems. Nucleic Acids Res. 2013;41:4336-43.

35. Mali P, Yang L, Esvelt KM, Aach J, Guell M, Dicarlo JE, et al. RNA-guided human genome engineering via Cas9. Science. 2013;339:823-7.

36. Hwang WY, Fu Y, Reyon D, Maeder ML, Tsai SQ, Sander JD, et al. Efficient genome editing in zebrafish using a CRISPR-Cas system. Nat Biotechnol. 2013;31:227-9.

37. Aparicio Tomás, Víctor de Lorenzo EM-G. CRISPR/Cas9-based counterselection boosts recombineering efficiency in Pseudomonas putida. Biotechnol J. 2017. https://doi.org/10.1002/biot.201700161.

38. Zetsche B, Gootenberg JS, Abudayyeh OO, Slaymaker IM, Makarova KS, Essletzbichler P, et al. Cpf1 is a single RNA-guided endonuclease of a Class 2 CRISPR-Cas system. Cell. 2015;163:759-71.

39. Makarova KS, Zhang F, Koonin EV. SnapShot: class 2 CRISPR-Cas systems. Cell. 2017;168(328-328):e1.

40. Martínez-Garćía E, Aparicio T, Goñi-Moreno A, Fraile S, De Lorenzo V. SEVA, 2.0: an update of the standard European vector architecture for de-/re-construction of bacterial functionalities. Nucleic Acids Res. 2015;43:D1183-9.

41. Winsor GL, Lam DKW, Fleming L, Lo R, Whiteside MD, Yu NY, et al. Pseudomonas genome database: improved comparative analysis and population genomics capability for Pseudomonas genomes. Nucleic Acids Res. 2011;39:596-600

42. Xiao A, Cheng Z, Kong L, Zhu Z, Lin S, Gao G, et al. CasOT: a genome-wide Cas9/gRNA off-target searching tool. Bioinformatics. 2014;30:1180-2.

43. Gruber S, Hagen J, Schwab H, Koefinger P. Reprint of "Versatile and stable vectors for efficient gene expression in Ralstonia eutropha $\mathrm{H} 16$ ". J Biotechnol. 2014;192:410-8.

44. Salis HM, Mirsky EA, Voigt CA. Automated design of synthetic ribosome binding sites to precisely control protein expression. Nat Biotechnol. 2010:27:946-50.

45. Jiang Y, Qian F, Yang J, Liu Y, Dong F, Xu C, et al. CRISPR-Cpf1 assisted genome editing of Corynebacterium glutamicum. Nat Commun. 2017;8:15179.

46. Pyne ME, Moo-Young M, Chung DA, Chou CP. Coupling the CRISPR/Cas9 system with lambda red recombineering enables simplified chromosomal gene replacement in Escherichia coli. Appl Environ Microbiol. 2015;81:5103-14

47. Li Y, Lin Z, Huang C, Zhang Y, Wang Z, Tang YJ, et al. Metabolic engineering of Escherichia coli using CRISPR-Cas9 meditated genome editing. Metab Eng. 2015;31:13-21.

48. Zhao D, Yuan $S$, Xiong B, Sun $H$, Ye L, Li J, et al. Development of a fast and easy method for Escherichia coli genome editing with CRISPR/Cas9. Microb Cell Fact. 2016:15:205

49. Choi KH, Kumar A, Schweizer HP. A 10-min method for preparation of highly electrocompetent Pseudomonas aeruginosa cells: application for DNA fragment transfer between chromosomes and plasmid transformation. J Microbiol Methods. 2006:64:391-7.

50. Li Q, Chen J, Minton NP, Zhang Y, Wen Z, Liu J, et al. CRISPR-based genome editing and expression control systems in Clostridium acetobutylicum and Clostridium beijerinckii. Biotechnol J. 2016;11:961-72.

51. Kehrenberg C, Catry B, Haesebrouck F, De Kruif A, Schwarz S. Novel spectinomycin/streptomycin resistance gene, aadA14, from Pasteurella multocida. Antimicrob Agents Chemother. 2005;49:3046-9.

52. Jin K, Zhou L, Jiang H, Sun S, Fang Y, Liu J, et al. Engineering the central biosynthetic and secondary metabolic pathways of Pseudomonas aeruginosa strain PA1201 to improve phenazine-1-carboxylic acid production. Metab Eng. 2015;32:30-8.

53. Wang Y, Zhang ZT, Seo SO, Lynn P, LuT, Jin YS, et al. Bacterial genome editing with CRISPR-Cas9: deletion, integration, single nucleotide modification, and desirable "clean" mutant selection in Clostridium beijerinckii as an example. ACS Synth Biol. 2016;5:721-32.

54. Wu J, Du G, Chen J, Zhou J. Enhancing flavonoid production by systematically tuning the central metabolic pathways based on a CRISPR interference system in Escherichia coli. Sci Rep. 2015;5:13477.

55. Wang M, Mao Y, Lu Y, Tao X, Zhu JK. Multiplex gene editing in rice using the CRISPR-Cpf1 system. Mol Plant. 2017;10:1011-3.

56. Kleinstiver BP, Tsai SQ, Prew MS, Nguyen NT, Welch MM, Lopez JM, et al. Genome-wide specificities of CRISPR-Cas Cpf1 nucleases in human cells. Nat Biotechnol. 2016:34:869-74.

57. Park J, Bae S, Kim JS. Cas-Designer: a web-based tool for choice of CRISPR-Cas9 target sites. Bioinformatics. 2015:31:4014.

58. Xie S, Shen B, Zhang C, Huang X, Zhang Y. SgRNAcas9: a software package for designing CRISPR sgRNA and evaluating potential off-target cleavage sites. PLoS ONE. 2014;9:1-9.

59. Slaymaker IM, Gao L, Zetsche B, Scott DA, Yan WX, Zhang F. Rationally engineered Cas9 nucleases with improved specificity. Science. 2016;351:84-8.

60. Stover CK, Pham XQ, Erwin AL, Mizoguchi SD, Warrener P, Hickey MJ, et al. Complete genome sequence of Pseudomonas aeruginosa PAO1, an opportunistic pathogen. Nature. 2000;406:959-64.

61. Silby MW, Cerdeño-Tárraga AM, Vernikos GS, Giddens SR, Jackson RW, Preston GM, et al. Genomic and genetic analyses of diversity and plant interactions of Pseudomonas fluorescens. Genome Biol. 2009;10:R51.

62. Poblete-Castro I, Becker J, Dohnt K, Dos Santos VM, Wittmann C. Industrial biotechnology of Pseudomonas putida and related species. Appl Microbiol Biotechnol. 2012:93:2279-90. 
63. Adams BL. The next generation of synthetic biology chassis: moving synthetic biology from the laboratory to the field. ACS Synth Biol. 2016;5:1328-30.

64. Nelson KE, Weinel C, Paulsen IT, Dodson RJ, Hilbert H, Martins dos Santos VAP, et al. Complete genome sequence and comparative analysis of the metabolically versatile Pseudomonas putida KT2440. Environ Microbiol. 2002;4:799-808.

65. Belda E, van Heck RGA, José Lopez-Sanchez M, Cruveiller S, Barbe V, Fraser $C$, et al. The revisited genome of Pseudomonas putida KT2440 enlightens its value as a robust metabolic chassis. Environ Microbiol. 2016;18:3403-24.

66. Park S-J, Choi J-S, Kim B-C, Jho S-W, Ryu J-W, Park D, et al. PutidaNET: interactome database service and network analysis of Pseudomonas putida KT2440. BMC Genom. 2009;10(Suppl 3):S18.

67. Nogales J, Palsson B $\varnothing$, Thiele I. A genome-scale metabolic reconstruction of Pseudomonas putida KT2440: iJN746 as a cell factory. BMC Syst Biol. 2008;2:79.

\section{Submit your next manuscript to BioMed Central and we will help you at every step:}

- We accept pre-submission inquiries

- Our selector tool helps you to find the most relevant journal

- We provide round the clock customer support

- Convenient online submission

- Thorough peer review

- Inclusion in PubMed and all major indexing services

- Maximum visibility for your research

Submit your manuscript at

www.biomedcentral com/submit 